became apparent that, although experimental systems using algae can be maintained in biological balance on a small scale, many difficulties would have to be overcome before they could be relied upon to provide human food and oxygen in a closed environment. Doubts were also expressed in a later paper by Mr. S. W. F. HaNSON (Ministry of Agriculture, Fisheries, and Food) whether an entirely algal diet would ever be acceptable from either the nutritional or the psychological point of view.

Returning to the psychological theme, Dr. K. F. JACKSON (R.A.F. Institute of Aviation Medicine) discussed some of the factors which impair human performance. Human performance in control, measured in terms of the accuracy with which pointers could be kept centred on a dial or an aircraft on its heading, could be affected by many variables. Some were obvious stresses applied, as it were, from without-such as environmental conditions affecting the equipment or the operator himself. Less well defined factors, including fatigue and motivation, caused changes in performance. Dr. Jackson's results related fluctuations in performance quantitatively to, for example, the time spent on an arduous task and to its complexity. The application of his work to astronautics would lie in determining the optimum "working conditions" and the best methods of presenting information and the means of control to the human pilot- "the most economical and versatile kind of controlling equipment that can be carried."

\section{Sensory Impoverishment}

Mr. C. Cunningham (Air Ministry) then discussed some effects of sensory impoverishment and sleep deprivation, both of which are predicted for space flight. In fact, feelings of mental pressure and perceptual disorder have already been reported by pilots in high altitude straight and level flight, or balloon ascents, where they are exposed to solitude and a reduced sensory input. Mr. Cunningham described experiments involving total sensory isolation, but compared the situation in space flight rather to certain patterns of political imprisonment. He argued that, in space flight, sensory impoverishment would be far from complete ; the pilot would have jobs to do and he would also be kept in touch with reality by the motive of self-preservation during his enforced confinement. The effects of "acute" sleep deprivation in some ways resembled those of alcoholic intoxication, but prolonged sleeplessness without ill effects was possible for those who had been trained to it.

\section{Environmental Hazards}

Among several papers dealing with physiological aspects of the sealed-cabin environment was one by Dr. G. PUGH (National Institute for Medical Research) which discussed possible economies in the requirements of oxygen and other equipment for short space flights lasting a few days. Payload, including structural weight, was at a very high premium in astronautics, and for this reason a low-pressure cabin was often envisaged. Acclimatization studies suggested that, not only could economies in oxygen and food be made by reducing dietary intake (to, say, 1,000 calories a day), but that preconditioned men could work at a reduced atmospheric oxygen tension. An equivalent altitude of $18,000 \mathrm{ft}$. $(5,500 \mathrm{~m}$.) was suggested. Dr. Pugh warned, however, that the cumulative hazard from endogenous carbon monoxide contaminating the cabin air would be intensified at a low oxygen tension. A further possibility of economy in space flight, which caught the imagination of the press, lay in the selection of very small people for crew members.

One of the anticipated hazards of space travel is heat stress, and in two detailed papers Flight Lieutenant $J$. BILlingham (R.A.F. Institute of Aviation Medicine) discussed problems of heat exchange between the man and his local environment in space. In many situations (for example, a re-entering vehicle heated during rapid penetra- tion of the atmosphere or a man in a "space suit" on the surface of the moon), it would be difficult to protect the man from excessive heat gain. The approach to this problem lay in the design of protective clothing and in artificial, positive cooling of the man in the vehicle.

Discussing the final and as yet ill-defined hazard of flight outside the atmosphere, Dr. A. E. Slater (British Interplanetary Society) described the finding of an intense belt of ionizing radiation girdling the earth. This zone, discovered by radiation monitors in the Explorer satellites, extended from, perhaps, 600 to 60,000 miles (966 to 96,600 $\mathrm{km}$.) above the earth and was believed to consist of highenergy protons arising from cosmic-ray bombardment of the upper atmosphere and trapped by the geomagnetic field. Since the radiation counters indicated that a lethal dose might be acquired in a few hours, the prospects of manned space flight looked gloomy. However, there was evidence that this intense radiation was a local phenomenonrestricted to certain geomagnetic latitudes, which-like the Sargasso Sea of yore-might be avoided by navigation.

Other contributions included papers by Dr. H. BJURSTEDT (Laboratory of Aviation and Naval Medicine, Stockholm) on the effects on pulmonary ventilation of prolonged exposure to positive $g$ loadings, and by Dr. A. S. PARKES, F.R.S., and Dr. AUdRey SMITH (National Institute for Medical Research) on space transport of life in the frozen state.

\section{New Appliances}

\section{A PRESSURE INJECTOR FOR USE IN ANGIOCARDIOGRAPHY AND AORTOGRAPHY}

Dr. J. N. Pattinson and Dr. Walter Somerville, of the Department of Radiodiagnosis and Cardiology, the Middlesex Hospital, London, write: The efficient injection of contrast medium is an important factor in the production of good-quality angiocardiograms. Our initial experience with simple manual injection soon revealed the shortcomings of this method and the need for a pressure device to ensure a rapid and safe delivery. Unassisted manual methods cannot achieve an adequate rate of injection through a cardiac

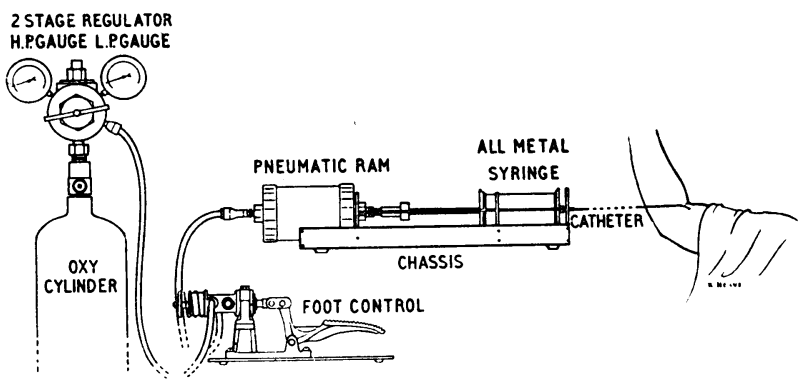

catheter or long "polythene" tube. The great advantage of selective angiography in its various forms, including thoracic aortography, further encouraged the development of the apparatus described below. At our suggestion $\mathrm{Mr}$. H. A. E. Talley desiøned a vacuum-operated device along the lines of that described by R. H. Morgan (Amer. J. Roentgenol., 1950, 64, 189). This proved unsuitable, and a change-over to positive-pressure actuation was made.

During the course of the last six years several minor modifications in the design have been made. The apparatus consists of a pneumatic ram mounted on a chassis which can hold an all-metal syringe of $25-, 50-$, or $100-\mathrm{ml}$. capacity. The ram is connected to a cylinder of compressed oxygen, air, or nitrogen through a two-stage variable pressureregulating valve fitted with an output pressure gauge and 
foot or hand control (see Diagram). When the latter is depressed the compressed gas passes to the ram, which applies a thrust to the piston of the syringe and empties it. The release of the foot or hand control immediately exhausts the gas in the ram to atmospheric pressure. In practice we have used only two gauge settings-one of 90-120 lb. per sq. in. (6.3-8.4 kg. per sq. cm.) for injection through a catheter, and the other of $25-35 \mathrm{lb}$. per sq. in. $(1.8-2.5 \mathrm{~kg}$. per sq. $\mathrm{cm}$.) for intravenous injection. The actual pressure applied to the ram is seven times that indicated on the gauge.

When employing selective angiocardiography a cardiac catheter of the largest bore possible is passed under fluoroscopic control through a peripheral vein into the heart. The catheter tip is placed in the superior or inferior vena cava, right or left atrium, right ventricle, or pulmonary artery, the exact site depending on the type of case under investigation. For thoracic aortography the catheter is inserted into the radial artery immediately distal to its origin, and is advanced into the ascending aorta so that its tip lies 2 in. $(5 \mathrm{~cm}$.) distal to the aortic valves. Alternatively, a polythene tube may be inserted percutaneously into brachial, carotid, or femoral arteries, and its tip advanced to the same site. The patient is then moved into position on the film changer and the catheter connected to the syringe on the injector chassis. On depressing the hand control, rapid injection at a predetermined rate is achieved. In general, injection rates of $20-30 \mathrm{ml}$. per second are employed. The accompanying Table illustrates the various injection times which can be achieved by the use of this apparatus.

\begin{tabular}{|c|c|c|c|c|c|c|c|c|c|}
\hline & \multicolumn{4}{|c|}{ Quantity of Contras } & \multicolumn{3}{|c|}{ Medium Injected } & \multirow{2}{*}{$\begin{array}{c}\begin{array}{c}\text { Size of } \\
\text { Cardiac } \\
\text { Catheter }\end{array} \\
10 \\
9\end{array}$} & \multirow{2}{*}{$\begin{array}{l}\begin{array}{c}\text { Injection } \\
\text { Time in } \\
\text { Seconds }\end{array} \\
2 \\
2 \frac{1}{2}\end{array}$} \\
\hline $70 n$ & & . & . & $\cdots$ & .. & . & .. & & \\
\hline 60 & $"$ & $\cdots$ & . & . & . & . & .. & 10 & $\begin{array}{l}2 \\
21\end{array}$ \\
\hline 50 & " & .. & $\cdots$ & . & . & $\cdots$ & . & $\begin{array}{r}10 \\
9 \\
8\end{array}$ & $\begin{array}{l}1 \frac{1}{4} \\
2 \\
2 \frac{1}{2}\end{array}$ \\
\hline 30 & •• & $\cdots$ & $\cdots$ & .. & $\cdots$ & $\cdots$ & $\cdots$ & 10,9, and 8 & $1-1 \frac{1}{2}$ \\
\hline 25 & " & . & . & . & $\cdots$ & . & . & 10,9, and 8 & $\frac{1}{2}$ \\
\hline 20 & " & .. & $\cdots$ & $\cdots$ & $\cdots$ & . & $\cdots$ & 9,8 , and 7 & $1-1 \frac{1}{2}$ \\
\hline 15 & $"$ & . & .. & . & .. & . & .. & 9 and 8 & $1^{\frac{1}{2}}$ \\
\hline
\end{tabular}

The above injection times are obtained with a gauge reading of 90-120 lb. per sq. in. (6.3-8.4 kg. per sq. cm.).

The use of the $50-\mathrm{ml}$. and $25-\mathrm{ml}$. syringes facilitates accurate measurement of the smaller doses of contrast medium required in children and infants. Furthermore, the lower bore/stroke ratio and reduced friction of the smaller syringes permit an adequate injection rate even through a No. 7 catheter, which may be the largest that can be employed in the very young patient.

The injector has been used over 350 times at the Middlesex Hospital and the London Chest Hospital for angiocardiography and thoracic aortography. The only untoward incident ascribable to the apparatus was the rupture of an arm vein on three occasions. These accidents were undoubtedly due to incorrect positioning of the metal cannula employed at the time. Such have not been experienced since the metal cannula was replaced by flexible tubing or cardiac catheter.

The advantages of the injector are the provision of rapid safe injection of contrast medium into the chambers of the heart or the great vessels, and simplicity in design and operation. It is cheap to buy and maintain, and has given satisfactory results over the past six years.

The apparatus is manufactured by the Talley Anaesthetic Equipment Ltd., 37a, New Cavendish Street, London, W.1. A succession of registrars and assistants have used the injector, and at their suggestion several modifications have been incorporated in the design.

\section{To-day's Drugs}

In this column we publish from time to time authoritative notes on a selection of drugs in current use.

Tenormal (Imperial Chemical Industries Ltd.).

Perolysen (May and Baker Ltd.).

These are preparations of pempidine tartrate $(1: 2: 2: 6: 6$ pentamethylpiperidine hydrogen tartrate) in tablets of 5 and $10 \mathrm{mg}$. Pempidine is a tertiary amine which lowers blood pressure by ganglion blockade. When given by mouth it is freely absorbed from the gut, and diffuses easily into tissues, readily crossing the blood-brain barrier. It thus closely resembles mecamylamine in its action, the most important difference being its quicker excretion. Thus, in contrast to mecamylamine, which is slowly excreted, acts for up to 12 hours, and is administered twice daily, pempidine acts for 6 hours and is usually given four times daily. The hypotensive action is usually observed within an hour.

Pempidine may prove to be an improvement on mecamylamine, for all the drug in the plasma is active, in contrast to mecamylamine, $25 \%$ of which is bound to plasma protein. Furthermore, the rapid excretion helps to prevent toxic accumulation of the drug. The excretion of both drugs is influenced by urinary $p \mathrm{H}$, the clearance being increased in acid urine and decreased in alkaline urine. But variation in excretion is less with pempidine, and its hypotensive effect is therefore less likely to be influenced by changes in acid-base balance than that of mecamylamine.

Treatment may be started with $2.5 \mathrm{mg}$. orally four times daily, increasing each dose by $2.5 \mathrm{mg}$. daily until the desired control of blood pressure is achieved. A smooth hypotensive effect throughout the day may be expected. The indications for pempidine are those applicable to ganglionblocking agents in general-severe, complicated, or malignant hypertension. The same side-effects, such as faintness, nausea, and constipation, must be watched for, though they may be less severe.

N.H.S. basic price : Tenormal and perolysen, 50 5-mg. tabs., 7s. 6d. ; 50 10-mg. tabs., 13s. 4d.

Compocillin-V Filmtabs (Abbott Laboratories Ltd.).These are tablets of the hydrabamine salt of phenoxymethylpenicillin (penicillin V). They are available as $125-\mathrm{mg}$. tablets $(200,000$ units) or 250-mg. tablets $(400,000$ units). The advantage of penicillin $\mathrm{V}$ is that it resists destruction by the hydrochloric acid in the stomach, and adequate blood levels can therefore be obtained when it is given by mouth. Further information about penicillin $\mathrm{V}$ was given in this column on August 16 (p. 445). It is a convenient means of administering penicillin in cases of infection by organisms sensitive to it.

Peganone (Abbott Laboratories Ltd.).-This is ethotoin (3-ethyl-5-phenylhydantoin) made up in tablets of $500 \mathrm{mg}$ It is of the same family of chemicals as phenytoin sodium, and is also closely related in its chemical formula to methoin (5-ethyl-3-methyl-5-phenylhydantoin). The hydantoins as a class are fairly well tolerated by mouth, and they have a specific anticonvulsant effect, the nature of which is unknown. Each chemical in this family has different toxic effects, though, apart from rare blood dyscrasias, they have not been severe. The present member of this family has been subjected to an extensive clinical trial ; no severe toxic reactions have so far been reported, and it does not appear to have any toxic effects not already encountered in its widely used predecessors. It is likely to be of use for major epilepsy, and for focal attacks, but not for the petit mal of childhood.

N.H.S. basic price: 100 tabs., 26s. 8d. 